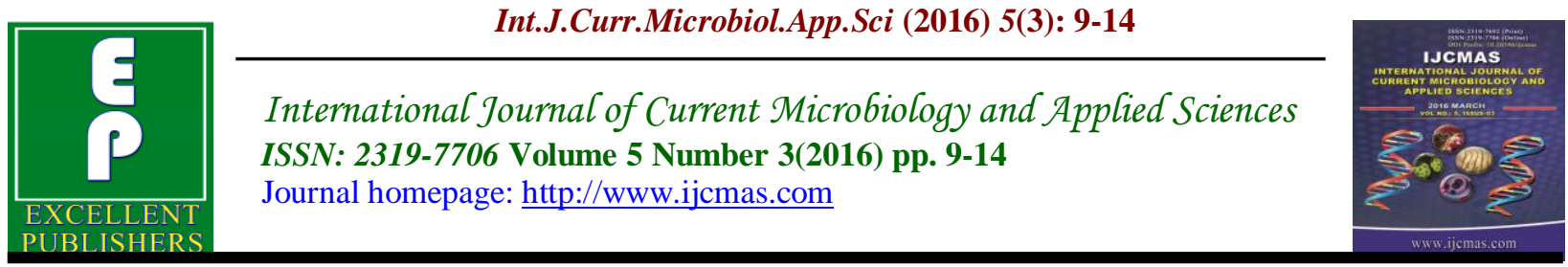

Research Article

http://dx.doi.org/10.20546/ijcmas.2016.503.002

\title{
Control of Cross Infection at Dental Clinic - A Survey
}

\author{
A. Santha Devy ${ }^{*}$, S. Rajkumari ${ }^{2}$ and A.N. Uma $^{3}$ \\ ${ }^{1}$ Department of Oral Pathology, Indira Gandhi Institute of Dental Sciences, \\ Pondicherry- 607 402, India \\ ${ }^{2}$ S.Rajkumari, Senior lecturer, Department of Oral Pathology, Sathyabama University Dental \\ College and Hospital, Sozhinganallur, Chennai - 600119, India \\ ${ }^{3}$ Genetic Division, Department of Anatomy, Mahatma Medical College and Research Institute, \\ Pondicherry- 607 402, India \\ *Corresponding author
}

Keywords

Awareness, Cross infection, Dental students, Infection control, Survey.

\section{Article Info}

Accepted:

07 February 2016

Available Online:

10, March 2016
A B S T R A C T

The concept of sterilization in the medical field had brought down the mortality and morbidity rate. Yet strict practice of disinfection remains questionable, either due to ignorance or negligence. Thus the study focused on the dental student population to assess their knowledge regarding the practice of infection control. The aim of the present study was to analyze the knowledge regarding control of cross infection among dental students of Pondicherry. It study design was a Questionnaire Survey and included 120 dental students from Indira Gandhi Institute of Dental Sciences, Pondicherry. A questionnaire related to cross infection from the dental healthcare worker to the patient, between the patients and from the patient to the dental healthcare worker, was distributed to the students and their level of knowledge and practice of infection control was assessed. In our study more than $90 \%$ of the students were aware about the need for medical history, Personal protective equipment, waste management, however the awareness regarding sterilization and disinfection was between 70 and $80 \%$. Thus, the dental students at Indira Gandhi Institute of Dental Sciences, Pondicherry reveal varying degree of knowledge regarding the infection control practices; however more awareness program is required to improve the quality of practice.

\section{Introduction}

The concept of sterilization in the medical field had brought down the mortality and morbidity rate. However strict practice of disinfection remains questionable, either due to ignorance or negligence. Unlike other body parts, oral cavity is in constant relation with atmosphere that contains variety of microbes. The dental unit and the associated treatments provide a rich source of microbial environment, establishing an equal chance for everyone at dental office to contract the infection. Hence in our study, we focused on the student population to assess their knowledge regarding the practice of 
infection control so as to assess if any additional attention in this aspect is required.

\section{Materials and Methods}

The study sample comprised of 120 dental students attending the outpatient department in Indira Gandhi Institute of Dental Sciences, Pondicherry. After obtaining approval from the institution, a questionnaire comprising of 19 self administered close ended questions was distributed to the students who were available on the day of survey. The participants were informed about the study in detail and were asked to mark the right answer whichever they felt was correct and were also asked not to get into any kind of discussions among their fellow mates. A sample questionnaire is given in Table 1.

\section{Results and Discussion}

The filled questionnaire was collected from the students and analyzed. Out of 120 students $99.16 \%$ reported that record of previous medical history is needed. Eighty percent of the students considered that washing hands is a must before and after wearing gloves and $94.16 \%$ before examining a patient.

About, 94.16\%, 95\%, 96.66\%, 96.66\%, $95 \%$ and $95.83 \%$ of the students considered Eye guard, Gowns/ aprons/lab coat, Gloves, Face mask, Head cap and all the above mentioned items were considered as Personal Protective Equipment for health care providers in the respective sequence. 96.66\% students believed that Gowns/ aprons and eye protection guards from the splashes and sprays of blood or body fluids. All the students thought that Gloves and mask prevents infection to health care provider while $82.5 \%$ of the students believed that they also prevent infection to the patient.
About $98.33 \%$ of the respondents opted for change of gloves after each patient. $80.83 \%$ of students mentioned that face mask should be changed between patients and $81.66 \%$ stressed upon the use of disposable paper towels. About 36.66\%, 14.16\%, 71.66\%, $96.66 \%$ reported that operative instruments that should be changed between the patients are in the order of burs, extraction instruments, hand piece, saliva ejectors. All of them deemed that autoclave is the best mode of sterilization while $85 \%$ believed in disinfecting solutions, $78.33 \%$ in hot water, $71.66 \%$ in detergent and $78.83 \%$ students believed in all of the above. $83.33 \%$ of the students considered that sterilized instruments should be maintained in plastic wrappings. When enquired about the equipments that require disinfection, Instruments tray and dental chair was given the maximum rating with $92.50 \%$ and $90 \%$ respectively, $87.50 \%$ to dental impressions, $80.83 \%$ to air/water syringe, $78.33 \%$ to focus light handle and extraction instruments, $76.66 \%$ to hand piece, $74.16 \%$ to spittoon and suction hose with least importance to dental burs taking up only $70.83 \%$.

Nearly $75 \%$ of the study population considered application of rubber dam is a must in conservative and endodontic dental procedures. Regarding the knowledge about wastes about $94.16 \%$ were aware about the categories of waste segregation. But the clarity about biomedical waste, anatomical waste and general waste was lagging at about $16.66 \%, 13.33 \%$, and $10 \%$ in the respective order. $94.16 \%$ of the students ensured that special container is a must for each category of waste.

Ninety percent of the students deemed regular cleaning of Wall, floor and carpets. $86.66 \%, 67.50 \%$ mandated that blood and body spills should be cleaned with 
disposable cloth and disinfectant respectively, $96.66 \%$ of the students responded positively to vaccination of all the health care personals regularly.

To attain best of prognosis in the course of disease process, practice of asepsis is mandatory. Microorganisms in the oral cavity are mostly normal commensals, which takes a lead role under favorable condition causing infection. Dental Health professionals constantly handle the most infectious saliva and blood (Emir et al., 2009), hence they must be adequately equipped to abstain from being infected. Before any treatment protocol is been carried out, a thorough medical records of the patient is mandatory to avoid unwanted consequences. In our study about $99 \%$ of dental students enquired about patient's previous medical history.

Before starting with any dental procedure (examination / non-surgical / surgical) it is compulsory to get rid of the colonizing micro flora that resides on the superficial layers of the skin, this can be accomplished by routine handwash with plain or antimicrobial soap and water or alcoholbased hand rub (William et al., 2003).

In this study $80 \%$ of the respondents adhered to the protocol of washing hands before and after wearing the gloves and $94.16 \%$ washed their hands before examining the patients, whereas a study by Abhinav Singh et al. (2011) only $39.2 \%$ of their respondents used washed their hands before and after patient examination.

The Centre for Disease Control (CDC) recommends that gloves, surgical mask, protective eyewear, protective clothing (e.g., gowns and jackets) be used as Personal Protective Equipments (PPE) for a dental professionals (William et al., 2003) especially when using high-speed rotary instruments (Mehrdad et al., 2009) which splashes blood and saliva all around. In the present study, around $95 \%$ of them reported to have adhered to the CDC guidelines of PPE including the usage of headcap, while a study on the Turkish population revealed $96.30 \%$ of the dentists preferred to use barrier techniques such as gloves, masks (Emir et al., 2009) and an another study showed that $67.5 \%$ of respondents always wore lab coat, $68.9 \%$ always used safety glasses, $41 \%$ reported to have used protective eyewear, $93.2 \%$ used facemask and almost $100 \%$ of respondents reported always to have worn gloves (Tracy et al., 2005).

A study by Abhinav Singh et al., reported that $69.8 \%$ of their study population used face mask and gloves as an infection control measure (William et al., 2003).

PPE benefits both the patient as well as the dentist and even if they are not able to do all that is enlisted in PPE, the dentist should at least change the gloves and facemask between the patients. In the present study $98 \%$ of the students were reported to change their gloves and only $80.83 \%$ changed the gloves and facemask between the patients, while a study revealed that $38.5 \%$, of the respondents changed face masks after each patient glove (Tracy et al., 2005). In a national survey of Canadian dentists, only $70 \%$ dentists used the basic barriers (gloves, masks, eye protection) (Jawdekar, 2013).

Apart from PPE the operative instruments also should be changed between the patients to avoid cross infection between the patients. Among the various categories of instruments $96.66 \%$ of them deemed the change of saliva ejectors with least importance (14.16\%) to extraction instruments. 
Table.1 Estimates of the Knowledge and Practice of Disinfection among Dental Students

\begin{tabular}{|c|c|c|}
\hline S.No. & \multicolumn{2}{|c|}{ Questions } \\
\hline 1. & \multicolumn{2}{|r|}{ Record of previous medical history is needed. } \\
\hline \multirow[t]{3}{*}{2.} & \multicolumn{2}{|r|}{ Washing hands is necessary } \\
\hline & a) & Before wearing gloves \\
\hline & b) & Before examining a patient \\
\hline \multirow[t]{7}{*}{3.} & \multicolumn{2}{|r|}{ Which of the following is Personal Protective Equipment in terms of health care provider } \\
\hline & a) & Eye guard \\
\hline & b) & Gowns/ aprons/lab coat \\
\hline & c) & Gloves \\
\hline & d) & Face mask \\
\hline & e) & Head cap \\
\hline & f) & All of the above \\
\hline 4. & \multicolumn{2}{|r|}{ Gowns/ aprons and eye protection guards from the splashes and sprays of blood or body fluids } \\
\hline 5. & \multicolumn{2}{|r|}{ Gloves and mask prevents infection to health care provider } \\
\hline 6. & \multicolumn{2}{|r|}{ Gloves and mask prevents infection to the patient } \\
\hline 7. & \multicolumn{2}{|r|}{ Change of gloves is warranted after each patient } \\
\hline 8. & \multicolumn{2}{|r|}{ Face mask should be changed between patients } \\
\hline 9. & \multicolumn{2}{|r|}{ Use disposable paper towel for placing the operative instruments } \\
\hline \multirow[t]{5}{*}{10.} & \multicolumn{2}{|r|}{ Operative instruments that should be changed between the patients } \\
\hline & a) & Burs \\
\hline & b) & Extraction instruments \\
\hline & c) & Hand pieces \\
\hline & d) & Saliva ejectors \\
\hline \multirow[t]{6}{*}{11.} & & Which one of the following is effective way of sterilization? \\
\hline & a) & Use of autoclave \\
\hline & b) & Disinfecting solutions \\
\hline & c) & Hot water \\
\hline & d) & Detergent \\
\hline & e) & All of the above \\
\hline 12. & \multicolumn{2}{|r|}{ Sterilized instruments should be maintained in plastic wrappings } \\
\hline \multirow[t]{12}{*}{13.} & \multicolumn{2}{|r|}{ Which of the following requires disinfection } \\
\hline & a) & Dental chair \\
\hline & b) & Air/Water syringe \\
\hline & c) & Spitoon \\
\hline & d) & Suction hose \\
\hline & e) & Hand piece \\
\hline & f) & Focus light handle \\
\hline & g) & Instruments tray \\
\hline & h) & Dental impressions \\
\hline & i) & Extraction instruments \\
\hline & j) & Surgical instruments \\
\hline & k) & Burs \\
\hline 14. & \multicolumn{2}{|r|}{ Application of rubber dam is a must in conservative and endodontic dental procedures. } \\
\hline 15. & \multicolumn{2}{|r|}{ Waste is divided into three categories; general, biomedical and pathological } \\
\hline 16. & \multicolumn{2}{|r|}{ Special container should be used for disposal of each category of waste } \\
\hline 17. & \multicolumn{2}{|r|}{ Wall, floor, carpets should be cleaned regularly } \\
\hline \multirow[t]{3}{*}{18.} & $\mathrm{Blo}$ & d/body spill should be cleaned with \\
\hline & a) & Disposable cloths \\
\hline & b) & Disinfection with disinfectant \\
\hline 19. & All & ealth care personnel should be vaccinated for communicable diseases regularly \\
\hline
\end{tabular}


It is impossible to use disposable instruments for every patient as they are not cost effective, however re-usage can be advocated if they are thoroughly cleaned and sterilized. According to $\mathrm{CDC}$, the operative instruments are categorized based on their penetration ability (Critical, Semi-critical, noncritical items) and the mode of sterilization varies for each group. Critical items that penetrates the hard and soft tissue must be sterilized by heat, Semi-critical items just the touch intact / non intact mucous membranes hence it should be heat sterilized or disinfected at a high level, noncritical items are those that just contacts the skin hence they should be disinfected. The equipments that undergo sterilization should be well packed (William et al., 2003). 94\% of the Kuwaiti dentists used autoclave for sterilization (Morris et al., 1996). Whatever is the category of the instrument, heat sterilization and disinfecting solutions remains the most effective method of sterilization. In our study $100 \%$ of the students opted for autoclave and $85 \%$ consider disinfecting solutions. About $83.33 \%$ warrants the need for maintaining the sterilized equipments in plastic wrappings.

With regards to disinfection at dental setup, $90 \%$ of the present study group stressed upon disinfecting the dental chair, $92.5 \%$ on instrument tray, $70-80 \%$ on air/water syringe, hand piece, suction hose, spittoon, focus light handle, dental impressions, burs and surgical instruments. In a previous study, $91.4 \%$ of the study group believed in disinfection of the dental chair, clinic, and dental office (Abhinav Singh et al., 2011). It has been reported that the major source of infection either bacterial or viral is through biofilms that form in the waterlines, suction pipes and hoses, hence these water connections must be regularly checked (William et al., 2003). Rubber dam application is also effective barrier in infection control.

The premises in which the dental procedure is been carried out also must be thoroughly cleaned regularly to get rid of infection at primary level. Around 91.66\% the participants in our study deemed that the floor, wall and carpets must be cleaned regularly.

Nearly $94.16 \%$ of the dental students were aware about the category of the waste materials and their management. However the awareness regarding blood/body spill was highly lacking.

Dental professionals handling sharp equipments are in constant contact with highly infectious blood and saliva. Hence prevention of communicable disease can be accomplished by prior vaccination. In our study $96.66 \%$ of the students deemed that all the dental health care professionals must be regularly immunized with appropriate vaccines. A study conducted in Ontario revealed that all of the dental students were aware about immunization (Mc Carthy et al., 2000).

In conclusion, the compliance regarding the knowledge of infection control is comparatively better than similar other studies that had been carried out, which clearly portrays the awareness towards the protocols available. However, it had not reached all of the student population. Though, the teaching curriculum includes effective training program, the implementation at the clinical setup is still deficient.

In the field of dentistry, it not only the dental professional being victimized by endangering infections like HIV but also the patients are vulnerable. So the program of 
infection control has to be stressed periodically and must be monitored by a governing body at each locality.

\section{References}

Abhinav, S., Bharathi, M.P., Ajay, B., Sudhanshu, S., Anshika, S., Amrita, G. 2011. Knowledge, attitudes, and practice regarding infection control measures among dental students in central India. J. Dent. Edu., 75(3): 421-427.

Emir, Y., Duygu, S., SevgiCanbaz, Y., Sinasi, S., Seda, C. 2009. A survey of cross-infection control procedures: knowledge and attitudes of Turkish dentists. J. Appl. Oral Sci., 17(6): 565-9.

McCarthy, G.M., Jonathan, E. Britton. 2000. A survey of final-year dental, medical and nursing students: occupational injuries and infection control. J. Can. Dent. Assoc., 66: 561.

Jawdekar, A.M. 2013. Infection control policy for dental practice: an evidence based approach. J. Contemp. Dent., 3(2): 82-86.

Mehrdad, A., Ojan, A. 2009. Infection control practices among dental professionals in Shiraz dentistry school, Iran. Arch Iranian Med., 12(1): 48-51.

Morris, E., Fuad, S.H., Abdulrazzak, AlNafisi, Sugathan, T.N. 1996. Infection control knowledge and practices in Kuwait - a survey on oral health care workers. Saudi Dent. J., 8(1): 45-54.

Tracy, B.K., Kathleen, B.M. 2005. A national survey of dental hygienists' infection control attitudes and practices. J. Dent. Hygiene, 79(2): 9298.

William, G.K., Amy, S.C., Jennifer, L.C., Jennifer, A.H., Kathy, J., Dolores, M.M. 2003. Centers for disease control and prevention. Guidelines for infection control in dental health-care settings - MMWR. 52.

\section{How to cite this article:}

Santha Devy, A., S.Rajkumari and Uma, A.N. 2016. Control of Cross Infection at Dental Clinic-A Survey. Int.J.Curr.Microbiol.App.Sci. 5(3): 9-14. doi: http://dx.doi.org/10.20546/ijcmas.2016.503.002 